\title{
FISH FAUNA STATUS OF THE NATURA 2000 SITES PROPOSED AS NEW OR FOR EXTENSION IN THE RIVERS SOMEŞ AND MUREŞ (ROMANIA)
}

\author{
Aurel NĂSTASE * and Vasile OŢEL ** \\ * Danube Delta National Institute for Research and Development, Babadag Street 165, Tulcea, \\ Romania, RO-820112, aurel.nastase@ddni.ro \\ ** Danube Delta National Institute for Research and Development, Babadag Street 165, Tulcea, \\ Romania, RO-820112, vasile_otel@yahoo.com
}

DOI: 10.1515/trser-2017-0021

KEYWORDS: fish ecological parameters, fish community interest, anthropogenic impact, Mureș and Someș rivers SCIs.

\section{ABSTRACT}

In autumn 2015 the fish fauna of river watersheds from six sites of community interest (SCI) Natura 2000 were investigated as follows: river Mureș (two SCIs) and Someș River in four SCIs from Someșul Mic, Someșul Mare and Someș. The researches were focused on the fish species of community interest (Annex II of Habitats Directive), its position in the ichthyocoenose (fish species richness, abundance and ecological parameters) and anthropogenic pressure. In investigated SCIs rivers researchers found 27 fish species including 11 species of community interest. IBI index shows moderate to good, or excellent levels of the evaluation integrity degree in fish fauna ecosystems.

ZUSAMMENFASSUNG: Zustand der Fischfauna in neu oder zur Erweiterung vorgeschlagenen Natura 2000-Gebieten der Flüsse Someș und Mureș im nördlichen und westlichen Grenzgebiet Rumäniens.

Im Herbst 2015 wurde die Fischfauna von sechs Fluss bezogenen Natura 2000Gebieten (SCI), zwei am Mureş und vier am Someș (Kleiner Someş, Großer Someş und vereinigter Someș-Fluss) untersucht. Die Forschungen waren ausgerichtet auf die Fischarten von gemeinschaftlichem Interesse (Anhang II der FFH-Richtlinie), ihre Stellung in der Fischzönose (Artenreichtum, Abundanz und ökologische Parameter) sowie den Einfluss menschlichen Druckes auf die Fischfauna. In den untersuchten Gebieten wurden 27 Fischarten festgestellt, darunter 11 Arten von gemeinschaftlichem Interesse. Der IBI-Index zeigt einen mäßigen bis guten, sogar bis hin zu einem exzellenten Integritätsgrad der Fischfauna und ihrer Ökosysteme.

REZUMAT: Starea faunei piscicole din situri Natura 2000 propuse ca noi sau spre extindere în râurile Someş şi Mureş, pe teritoriul României.

În toamna anului 2015 a fost investigată fauna piscicolă din şase SCI-uri după cum urmează: râurile Mureș (două SCI-uri) și Someș (în patru SCI-uri pe Someșul Mic, Someșul Mare și Someșul Unit). Cercetările au fost concentrate asupra speciilor de pești de interes comunitar (Anexa II din Directiva Habitate), poziția lor în ihtiocenoză (bogăția speciilor de pești, abundența speciilor și câțiva parametrii ecologici) totodată și influența presiunii antropice. În SCI-urile investigate au fost găsit 27 specii de pești, inclusiv 11 specii de interes comunitar. Indicatorul IBI arată un nivel moderat până la bun spre excelent privitor la evaluarea gradului de integritate în ecosistemele faunei piscicole. 


\section{INTRODUCTION}

Before 2015, Romanian Ministry of Environment promoted 273 sites of community interest (SCIs) that took 13.21\% from Romanian territory in 2007. After revision in 2011 more sites were enlarged, and new sites were designated thus reaching to 383 SCIs. Therefore, SCI's now occupy 16.76\% of Romanian territory (Brânzan, 2013; Oțel and Năstase, 2010; Năstase and Oțel, 2016) according to Romanian Order 2,387/2011 modifying Order 1964/2007. Therefore, at the end of 2015 a total number of 434 SCIs or others ones enlarged were reported to the EU. The total surface of SCIs and SPAs cover about 25\% of Romania.

A reference list of fish species of community interest from Romania contains 26 types of fish (Tatole et al., 2009) in accordance with Habitats Directive no. 92/43/1992 and Romanian Law 49/2011 (completing Romanian OUG 57/2007).

Previously, Standard List Form of Natura 2000 in studied SCIs (ROSCI0367, ROSCI0368, ROSCI0394) contained nine species of community interest as follows: Aspius aspius, Barbus meridionalis, Cobitis taenia, Gobio albipinnatus, Gobio kessleri, Gobio uranoscopus, Rhodeus sericeus amarus, Sabanejewia aurata, Zingel streber (in accordance with the nomenclature adopted under the Habitats Directive species lists). We must emphasise that current nomenclature is modified to eight fish species, except Zingel streber. European Union requested for Aspius aspius, Gobio albipinnatus and Rhodeus amarus related with the mammal Lutra lutra all considered In Mod (In Moderate) to design new SCIs, or enlarge some pre-existing SCIs on the Mureș and Someș rivers.

The total number of fish species present in the basin of the river Someş is 62 (49 of them native and 13 introduced) (Bănărescu et al., 1999). There is strongly anthropogenic impact mostly located in Someşul Mic and Someş rivers downstream Baia Mare locality. There are also major changes in fish assemblages because of water polluation confirmed in the Hungarian section of Someş River (Antal et al., 2013).

From Mureş River were captured 56 fish species, but the pollution led to negative impacts and led to the loss of some species (Nalbant, 1993, 1994, 1995; Köhler et al., 2005, 2007; Curtean-Bănăduc et al., 2007, 2017; Sandu et al., 2008).

The new proposals for SCI lists were completed for the first time with native fish species in the studied area. Enlarged SCIs added new fish species of community interest.

\section{MATERIAL AND METHODS}

Depending on the morphology of water bodies and the flow velocity, we used different gear for fish sampling as follows: an inflatable boat of two-person, Nordic gillnets (with 12 panels $2.5 \mathrm{~m}$ each panel, with multiple meshes size six-55 mm), also electric fishing device SAMUS 725MP with accumulator $12 \mathrm{~V}$ and 5-60 Amps output $600 \mathrm{~W}$. Also angling and data from local fishermen were used. Electric fishing was carried out on day and gillnets fishing on night (12 hours stationary). It was assessed the presence of community interest species (Annex II/Habitats Directive no. 92/43/EC), quantitative structure (numerical abundance, biomass), specimen dimensions, analytical and synthetically ecological indicators, overall status of aquatic habitats in terms of existing anthropogenic pressures.

The camps were installed as close as to the banks of water bodies, about the middle sites. At each site, we performed fishing at least one point (approximately to the middle of SCI sites) or in two points each with a length of approximate $100 \mathrm{~m}$ river beds (according to the methodology specified in the Habitats Directive no. 92/43/EC).

The catch was sorted by species (fish identification according to the latest systematic reviews after Bănărescu (1964) with updates after Bănărescu (1994, 2004), Kottelat (1997), Kottelat and Freyhof (2007), Froese and Pauly (2016); weighing and measuring of lengths are 
performed. The abundances and biomass were determinate to each species and site, in order to find the status of species in the fish community. After measurements, the remaining individuals were released into the river. The human impact was also assessed. Few specimens were collected and preserved in alcohol for species that we had doubts of correct identification.

It was assessed fish fauna community, especially presence of community interest species, quantitative structure (numerical abundance), ecological indices $(\mathrm{D}=$ dominance, $\mathrm{C}=$ constancy, $\mathrm{W}=$ ecological significance, table 1), IBI (biological integrity index in tables 2 and 3).

Table 1: Frequency ( $\mathrm{C}=$ constancy), dominance (D) and ecological significance (W) classification (Odum, 1975; Schwerdtfeger, 1975; Botnariuc and Vădineanu, 1982; Muhlenberg, 1993; Gomoiu and Skolka, 2001; Șindrilariu et al., 2002; Sârbu and Benedek, 2004).

\begin{tabular}{|c|c|c|c|c|c|}
\hline \multicolumn{2}{|c|}{ Dominance (D) } & \multicolumn{2}{c|}{ Constancy (C) } & \multicolumn{2}{c|}{ Ecological significance (W) } \\
\hline Class & $\%$ & Class & $\%$ & Class & $\%$ \\
\hline sporadic D1 & $<1$ & very rare & C1 $=0-10$ & accidental & W1 $<0.1$ \\
\hline subrecedent D2 & $1\left(2^{0}\right)-<$ & rare & C2 $=10.1-25$ & accessory & W2 $=0.1-1$ \\
\hline recedent D3 & $2\left(2^{1}\right)-<$ & widespread & C3 $=25.1-45$ & associate & W3 =1-5 \\
\hline subdominant D4 & $4\left(2^{2}\right)-<$ & frequent & C4 $=45.1-70$ & complementa & W4 = 5-10 \\
\hline dominant D5 & $8\left(2^{3}\right)-16$ & very frequent & C5 $=70.1-100$ & characteristic & W5 =10- \\
\hline eudominant D6 & $>16\left(2^{4}\right)$ & & & main, leading & W6 $>20$ \\
\hline
\end{tabular}

Table 2: Criteria of fish determining IBI (biological integrity index) (Miller, 1985; Karr et al., 1986; Battes, 1991).

\begin{tabular}{|c|c|c|c|c|}
\hline \multirow[b]{2}{*}{$\begin{array}{l}\text { Parameters } \\
\text { categories }\end{array}$} & \multirow[b]{2}{*}{ Parameter } & \multicolumn{3}{|c|}{ Evaluation integrity class } \\
\hline & & $\begin{array}{c}5 \\
\text { (abund.) }\end{array}$ & $\begin{array}{c}3 \\
\text { (const.) }\end{array}$ & $\begin{array}{c}1 \\
\text { (rare) }\end{array}$ \\
\hline \multirow{7}{*}{$\begin{array}{l}\text { Composition } \\
\text { and } \\
\text { abundance of } \\
\text { species }\end{array}$} & 1. Total number of fish species & $>90 \%$ & $50-90 \%$ & $<50 \%$ \\
\hline & 2. Total number of Cyprinidae sp. & $>45 \%$ & $20-45 \%$ & $<20 \%$ \\
\hline & 3. Total number of Salmonidae sp. & $>5 \%$ & $1-5 \%$ & $<1 \%$ \\
\hline & 4. Others fish sp. & $>20 \%$ & $5-20 \%$ & $<5 \%$ \\
\hline & 5. Total number of $n$ & $>68 \%$ & $35-67 \%$ & $<34 \%$ \\
\hline & 6. Total number of $\mathrm{I}$ & $<1 \%$ & $1-10 \%$ & $>10 \%$ \\
\hline & 7. Total no. of disa & $<1 \%$ & $1-10 \%$ & $>10 \%$ \\
\hline \multirow{4}{*}{$\begin{array}{l}\text { Composition } \\
\text { of the food } \\
\text { fish } \\
\text { populations }\end{array}$} & 8. Proportion (\%) of zoobentophagous & $>45 \%$ & $20-45 \%$ & $<20 \%$ \\
\hline & 9. \%of carnivore sp. & $>5 \%$ & $1-5 \%$ & $<1 \%$ \\
\hline & 10.\% of carnivore & $<20 \%$ & $20-45 \%$ & $>45 \%$ \\
\hline & $11 . \%$ of & $<25 \%$ & $25-50 \%$ & $>50 \%$ \\
\hline \multirow{4}{*}{$\begin{array}{l}\text { Stock and } \\
\text { general state } \\
\text { of fish } \\
\text { populations }\end{array}$} & $\begin{array}{l}\text { 12. Numerical Stock (ex./100 } \mathrm{m}^{2} \text { ) } \\
\text { (ex./100 m linear/collectors) }\end{array}$ & $\begin{array}{l}>100 \mathrm{ex} \\
(>20 \mathrm{ex})\end{array}$ & $\begin{array}{l}10-100 \\
(5-20)\end{array}$ & $\begin{array}{l}<10 \\
(<5)\end{array}$ \\
\hline & $\begin{array}{l}\text { 13. Gravimetrical Stock (g/100 m²) } \\
\text { (g/100 m linear/collectors) }\end{array}$ & $\begin{array}{l}>1000 \mathrm{~g} \\
(>5000 \mathrm{~g})\end{array}$ & $\begin{array}{l}100-1000 \\
(500-5000)\end{array}$ & $\begin{array}{l}<10 \\
(<5)\end{array}$ \\
\hline & 14. Proportion of hybrid individuals & $0 \%$ & $0-1 \%$ & $>1 \%$ \\
\hline & 15. Proportion of ill individuals & $0 \%$ & $0-1 \%$ & $>1 \%$ \\
\hline
\end{tabular}


Table 3: Framing levels of the evaluation integrity degree in fish ecosystems (Miller, 1985; Karr et al., 1986; Battes, 1991).

\begin{tabular}{|c|l|c|c|c|c|}
\hline No. & Appreciation & Score & & & $\begin{array}{c}\text { Evaluation } \\
\text { integrity class }\end{array}$ \\
\hline & & Small rivers & \multicolumn{2}{|c|}{ Medium and big rivers and reservoirs } \\
\hline & & (Miller, 1985) & (Karr et al., 1986) & (Battes, 1991) & \\
\hline 1. & Excellent & $37-40$ & $57-60$ & $70-75$ & I \\
\hline 2. & Excellent-good & $34-36$ & $53-56$ & $66-69$ & II \\
\hline 3. & Good & $30-33$ & $48-52$ & $59-65$ & III \\
\hline 4. & Moderate-good & $28-29$ & $45-47$ & $55-58$ & IV \\
\hline 5. & Moderate & $23-27$ & $39-44$ & $47-54$ & V \\
\hline 6. & Poor-moderate & $21-22$ & $36-38$ & $43-46$ & VI \\
\hline 7. & Poor & $16-20$ & $28-35$ & $35-42$ & VII \\
\hline 8. & Poor-very low & $12-15$ & $24-27$ & $20-34$ & VIII \\
\hline 9. & Very low & $<12$ & $<23$ & $<20$ & IX \\
\hline
\end{tabular}

The research was conducted during September-October 2015 in six Natura 2000 sites: two SCIs in Mureș River (ROSCI0367 Mureșul between Morești and Ogra and ROSCI0368 Mureș River between Reghin and Deda) and four SCIs in Someș River (Someșul Mic River ROSCI0394 near Gherla and Miniu Gherlii localities; lower Someș River - ROSCI0435 Ardusat locality to Romania-Hungary border; Someș River between Rona-Țicău ROSCI0436; and Someșul Mare River - ROSCI0437 Someș between Mica and Beclean) (Fig. 1).

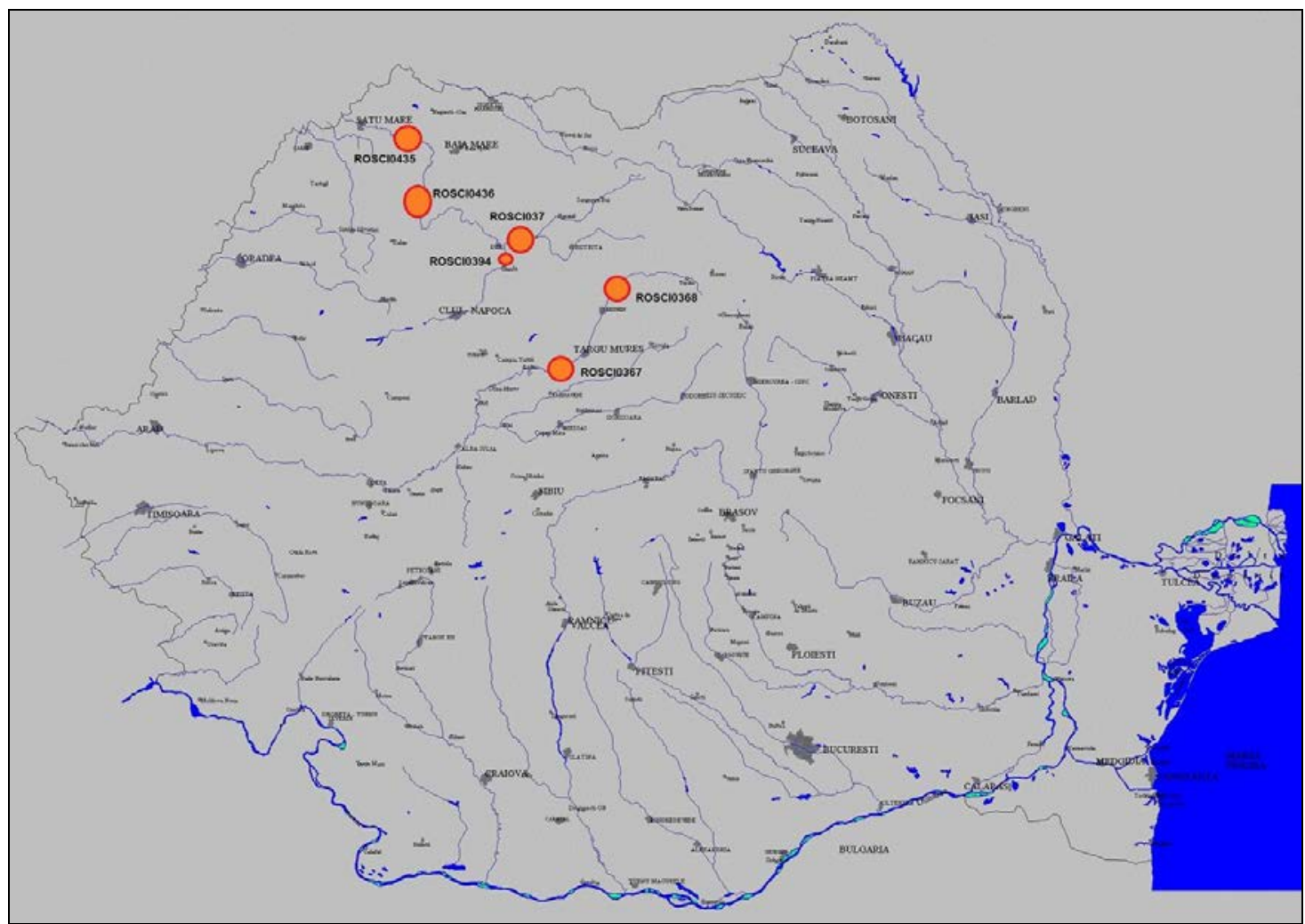

Figure 1: Overview of the project region, localisation of the SCIs points and SCIs codes. 


\section{RESULTS AND DISCUSSION}

In autumn of 2015 were captured 27 fish species including 11 ones of community interest, from these 11 species three are new added in Standard Form List N2000 of studied SCIs, meaning Hucho hucho, Cottus gobio and Zingel zingel, but uncaptured was one species in 2015 Zingel streber (Tab. 4).

We have to emphasis the first recording of more specimens of Neogobius fluviatilis (monkey goby) in Someșul Mare River upstream Dej locality, after first record of species in Someș River in 2014 downstream Dej locality (Cocan et al., 2014), confirming the hypotheses that this species is in a continuous expansion in Danube Basin. We also captured many $N$. fluviatilis individuals in Someș River downstream Dej locality, till to Romania-Hungary border.

Regarding fish abundance percentage in studied SCIs are presented in figures 2-7, with black column for fish species community interest and greyish column for others captured fish species.

Concerning ecological significance (Tabs. 5 and 6) Alburnus alburnus is main species in plain area of mostly studied SCIs (except Mureș River Reghin-Deda sector), but in submontain area (in Mureș River in Reghin-Deda sector) Alburnoides bipunctatus is main, dominant species. After these species, follows characteristic or complementary species like Squalius cephalus, Rhodeus amarus, Gobio albipinnatus and Barbus meridionalis with some differences between sites.

Table 4: Fish species richness captured in six Romanian SCIs from Someș and Mureș rivers in 2015 ( 1 = present species, bolded are fish species community interest).

\begin{tabular}{|c|c|c|c|c|c|c|c|c|}
\hline \multirow[b]{2}{*}{ No. } & \multirow[t]{2}{*}{ Species/Sector } & \multirow{2}{*}{$\begin{array}{c}\begin{array}{c}\text { Someşul } \\
\text { Mic } \\
\text { River }\end{array} \\
\frac{\pi}{\square} \\
\text { 志 }\end{array}$} & \multirow{2}{*}{ 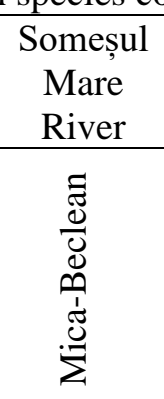 } & \multicolumn{3}{|c|}{$\begin{array}{c}\text { Someș } \\
\text { River }\end{array}$} & \multicolumn{2}{|c|}{$\begin{array}{l}\text { Mureș } \\
\text { River }\end{array}$} \\
\hline & & & & 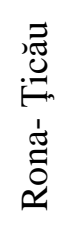 & 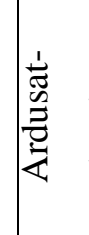 & 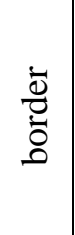 & 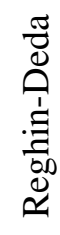 & 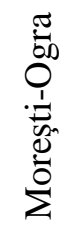 \\
\hline 1. & Alburnoides bipunctatus & 1 & 1 & 1 & & 1 & 1 & 1 \\
\hline 2. & Alburnus alburnus & 1 & 1 & 1 & & 1 & 1 & 1 \\
\hline 3. & Ballerus sapa & & & & & 1 & & \\
\hline 4. & Barbatula barbatula & & & & & & 1 & \\
\hline 5. & Barbus barbus & & 1 & 1 & & 1 & & 1 \\
\hline 6. & Barbus meridionalis & 1 & 1 & 1 & & & 1 & \\
\hline 7. & Carassius gibelio & & & 1 & & & & \\
\hline 8. & Chondrostoma nasus & 1 & 1 & 1 & & & 1 & \\
\hline 9. & Cobitis taenia & & & & & & 1 & 1 \\
\hline 10. & Cottus gobio & & & & & & 1 & \\
\hline 11. & Gobio gobio & & 1 & & & & 1 & 1 \\
\hline 12. & Gobio uranoscopus & & & & & & 1 & \\
\hline 13. & Hucho hucho & & & & & & 1 & \\
\hline
\end{tabular}


Table 4 (continued): Fish species richness captured in six Romanian SCIs from Someș and Mureș rivers in 2015 ( 1 = present species, bolded are fish species community interest).

\begin{tabular}{|c|c|c|c|c|c|c|c|c|}
\hline \multirow[b]{2}{*}{ No. } & \multirow[t]{2}{*}{ Species/Sector } & \multirow{2}{*}{$\begin{array}{c}\begin{array}{c}\text { Someșul } \\
\text { Mic } \\
\text { River }\end{array} \\
\\
\frac{\pi}{\frac{\pi}{4}}\end{array}$} & \multirow{2}{*}{ 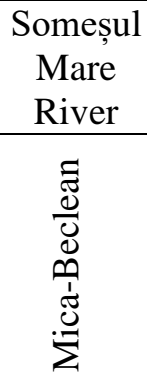 } & \multicolumn{3}{|c|}{$\begin{array}{c}\text { Someș } \\
\text { River }\end{array}$} & \multicolumn{2}{|c|}{$\begin{array}{c}\text { Mureș } \\
\text { River }\end{array}$} \\
\hline & & & & 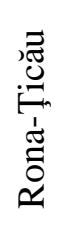 & 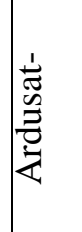 & 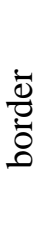 & 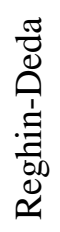 & 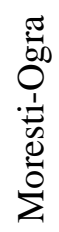 \\
\hline 14. & Lepomis gibbosus & 1 & & & & & & \\
\hline 15. & Aspius aspius & 1 & 1 & 1 & 1 & & 1 & 1 \\
\hline 16. & Neogobius fluviatilis & & 1 & 1 & 1 & & & \\
\hline 17. & Perca fluviatilis & & & 1 & & & & 1 \\
\hline 18. & Pseudorasbora parva & 1 & & 1 & 1 & & & \\
\hline 19. & Rhodeus amarus & 1 & 1 & 1 & 1 & & 1 & 1 \\
\hline 20. & Gobio kessleri & & 1 & 1 & & & & \\
\hline 21. & Gobio albipinnatus & 1 & 1 & 1 & 1 & & 1 & 1 \\
\hline 22. & Rutilus rutilus & 1 & & 1 & 1 & & & 1 \\
\hline 23. & Sabanejewia aurata & & 1 & 1 & & & 1 & \\
\hline 24. & Silurus glanis & & & & 1 & & & \\
\hline 25. & Squalius cephalus & 1 & 1 & 1 & 1 & & 1 & 1 \\
\hline 26. & Vimba vimba & & 1 & 1 & 1 & & & 1 \\
\hline 27. & Zingel zingel & & & & 1 & & & \\
\hline & TOTAL & 11 & 14 & 17 & 12 & & 15 & 12 \\
\hline & Fish community interest & 4 & 6 & 6 & 4 & & 8 & 4 \\
\hline
\end{tabular}

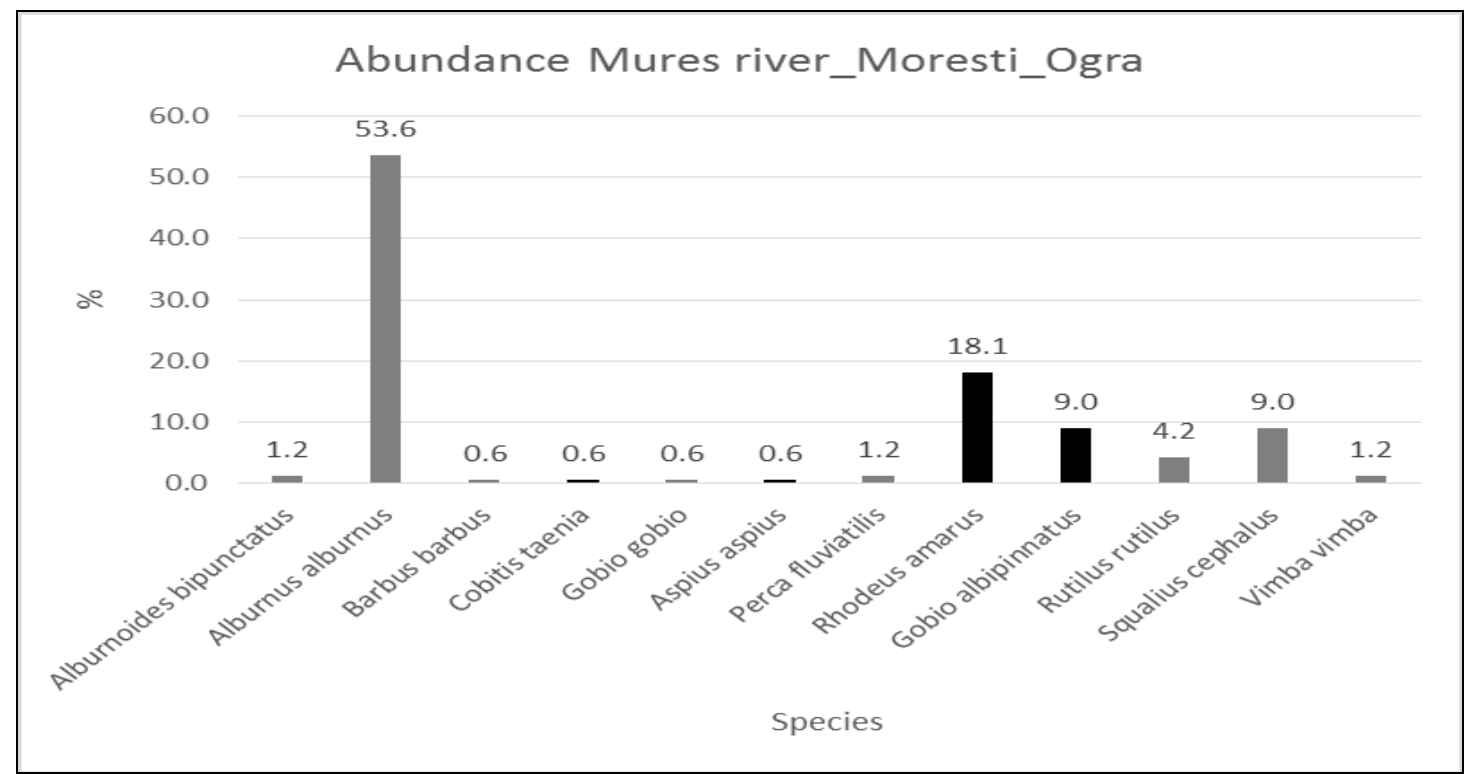

Figure 2: Fish abundance (percent) from Mureș River in ROSCI0367 (Mureș between Morești and Ogra). 


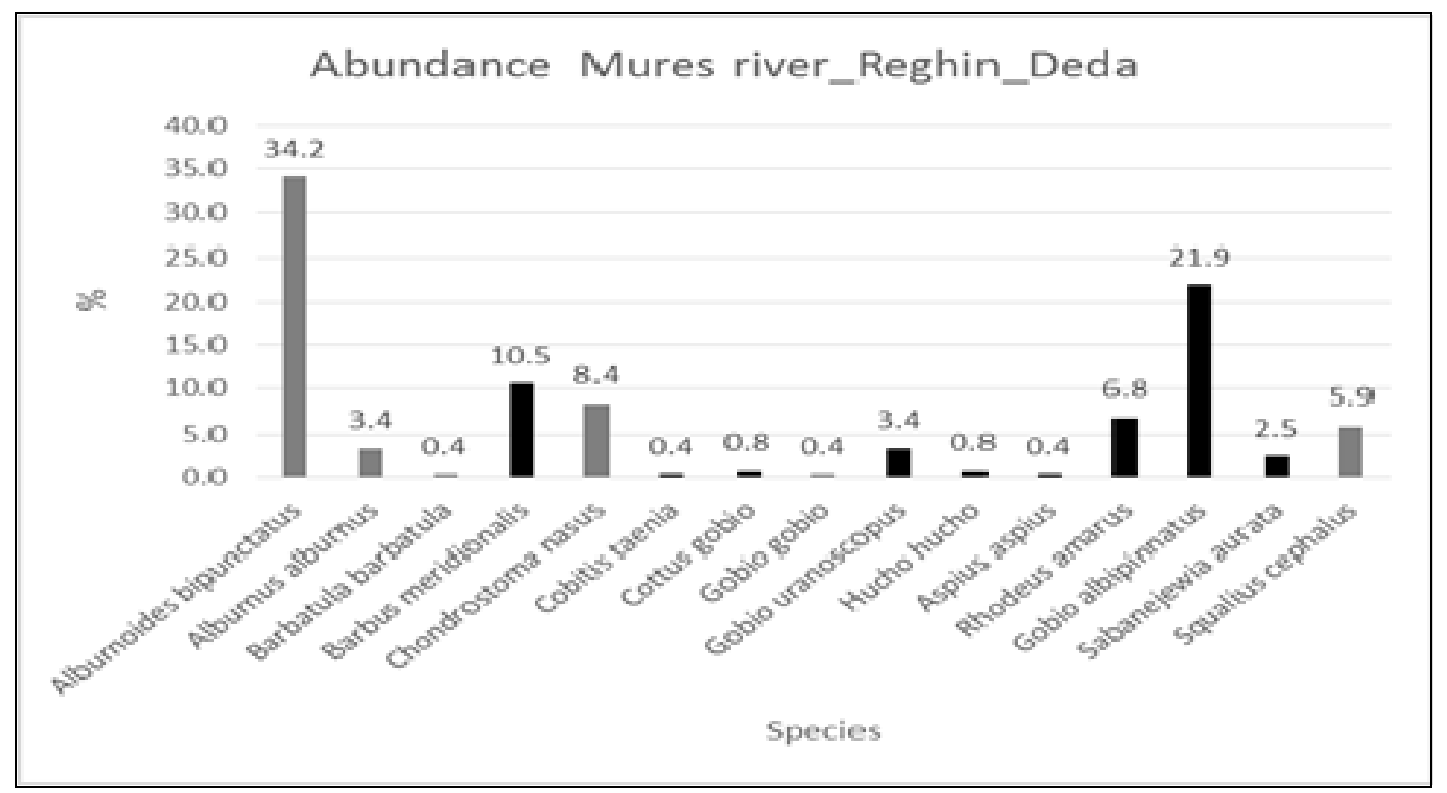

Figure 3: Fish abundance (percent) from Mureș River in ROSCI0368 (Mureș between Reghin-Deda).

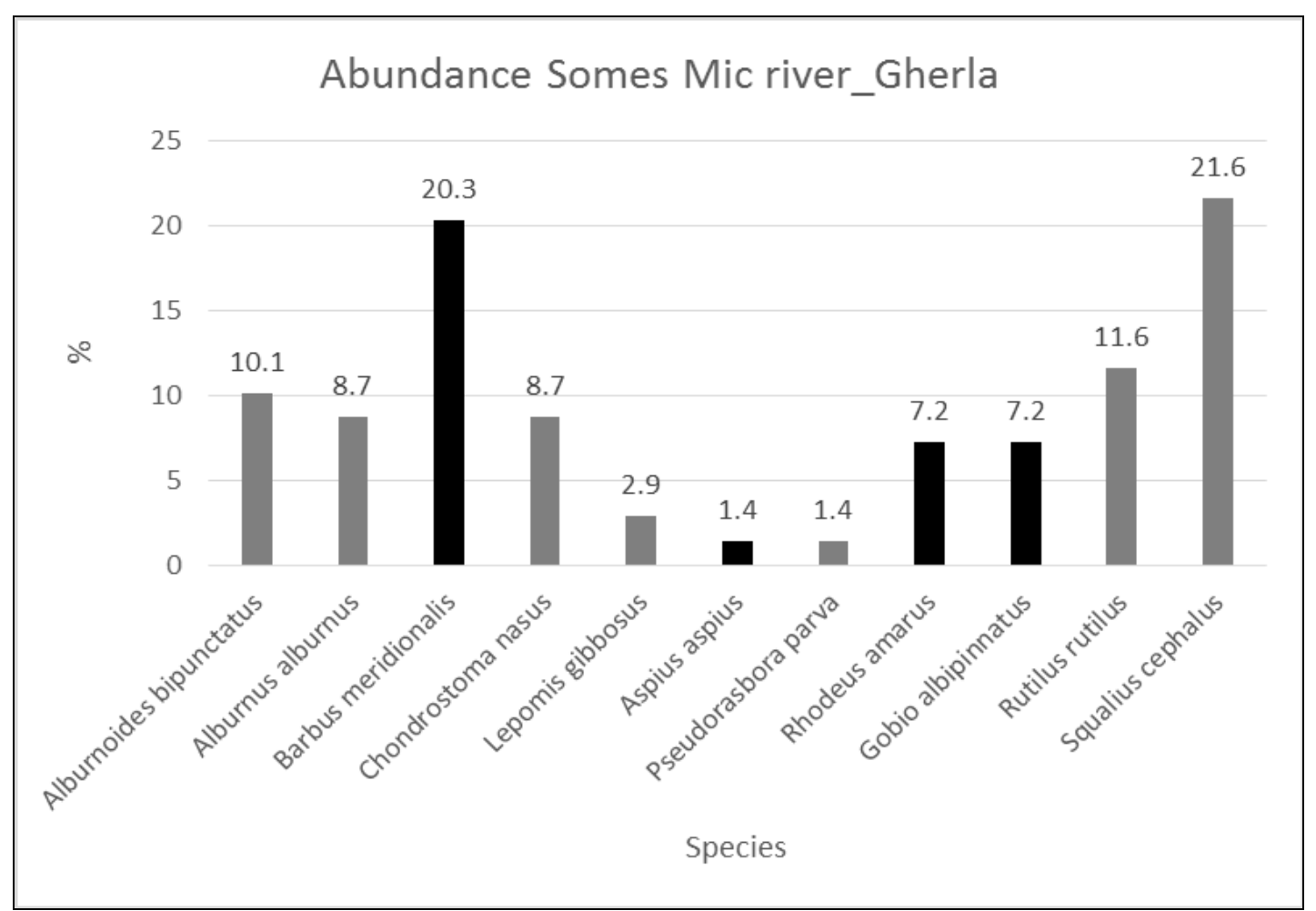

Figure 4: Fish abundance (percent) from Someș Mic River in ROSCI0394 (Someşul Mic). 


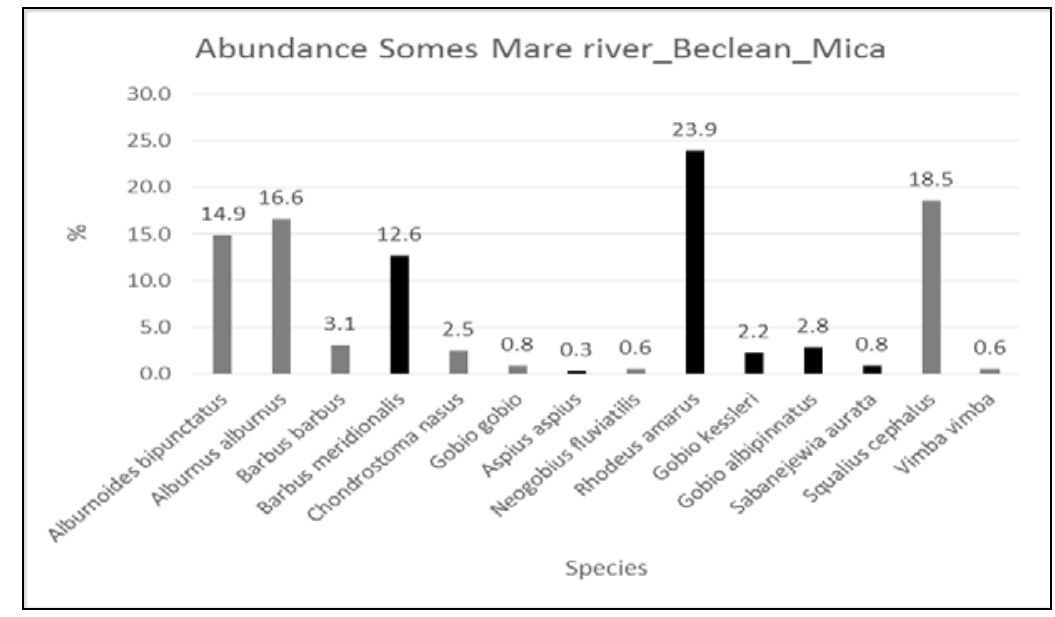

Figure 5: Fish abundance (percent) from Someș Mare River in ROSCI0437 (Someșul Mare between Mica-Beclean).

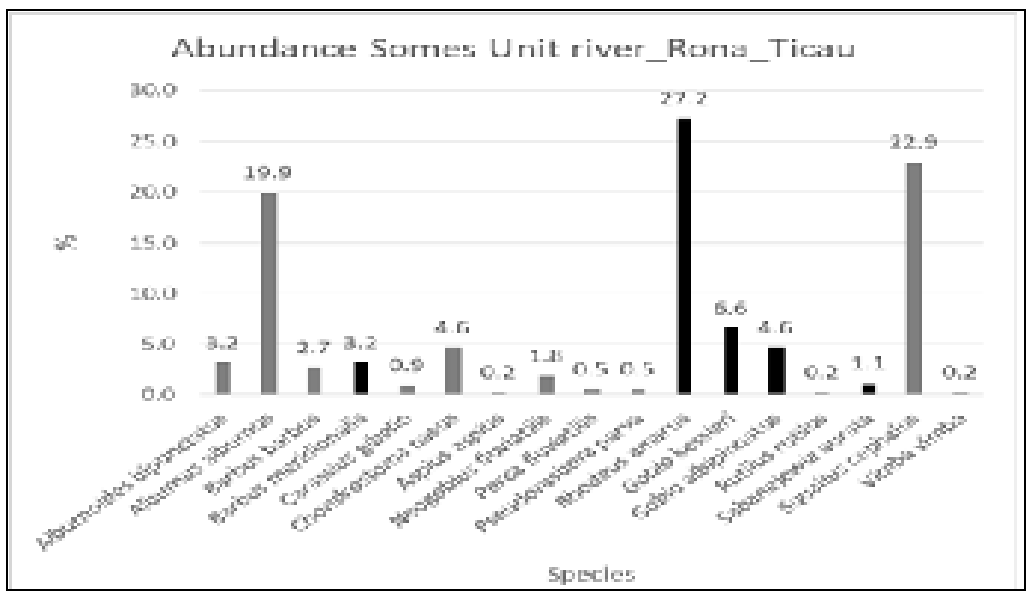

Figure 6: Fish abundance (percent) from United Someș River in ROSCI0436

(Someș between Rona-Țicău).

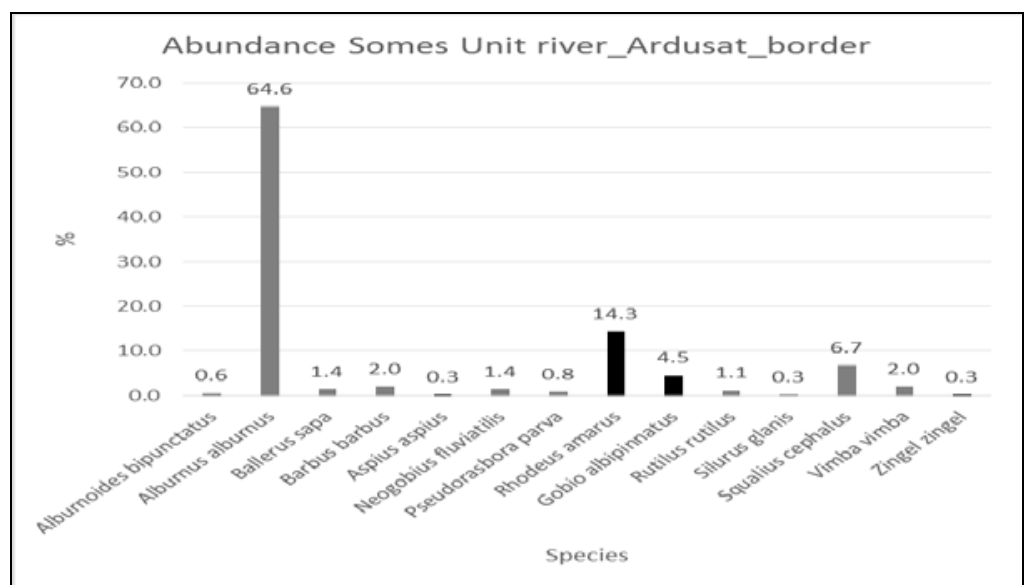

Figure 7: Fish abundance (percent) from Someș River in ROSCI435 (lower Someș River) Ardusat locality to border. 
Table 5: Ecological significance from three extended SCIs in 2015.

\begin{tabular}{|c|c|c|c|c|c|c|c|c|c|}
\hline \multirow{3}{*}{ Species } & \multicolumn{3}{|c|}{ Someșul Mic River } & \multicolumn{6}{|c|}{ Mureș River } \\
\hline & \multirow[b]{2}{*}{$\begin{array}{c}\mathrm{D} \\
\text { class }\end{array}$} & \multirow[b]{2}{*}{$\begin{array}{c}\mathrm{C} \\
\text { class }\end{array}$} & \multirow[b]{2}{*}{$\begin{array}{c}\text { W } \\
\text { class }\end{array}$} & \multicolumn{3}{|c|}{ Reghin-Deda } & \multicolumn{3}{|c|}{ Morești-Ogra } \\
\hline & & & & $\begin{array}{c}\text { D } \\
\text { class }\end{array}$ & $\begin{array}{c}\text { C } \\
\text { class }\end{array}$ & $\begin{array}{c}\text { W } \\
\text { class }\end{array}$ & $\begin{array}{c}\mathrm{D} \\
\text { class }\end{array}$ & $\begin{array}{c}\mathrm{C} \\
\text { class }\end{array}$ & $\begin{array}{c}\mathrm{W} \\
\text { class }\end{array}$ \\
\hline Alburnoides bipunctatus & D3 & $\mathrm{C} 2$ & W2 & D6 & $\mathrm{C} 5$ & W6 & $\mathrm{D} 2$ & $\mathrm{C} 2$ & W2 \\
\hline Alburnus alburnus & D3 & $\mathrm{C} 3$ & W3 & $\mathrm{D} 2$ & $\mathrm{C} 2$ & W2 & D6 & C5 & W6 \\
\hline \multicolumn{10}{|l|}{ Ballerus sapa } \\
\hline Barbatula barbatula & & & & D1 & C1 & W1 & & & \\
\hline Barbus barbus & & & & & & & D1 & C1 & W1 \\
\hline Barbus meridionalis & D6 & $\mathrm{C} 4$ & W5 & D6 & $\mathrm{C} 4$ & W5 & & & \\
\hline \multicolumn{10}{|l|}{ Carassius gibelio } \\
\hline Chondrostoma nasus & D3 & $\mathrm{C} 2$ & W2 & $\mathrm{D} 2$ & $\mathrm{C} 4$ & W3 & & & \\
\hline Cobitis taenia & & & & D1 & C1 & W1 & D1 & C1 & W1 \\
\hline Cottus gobio & & & & D1 & C1 & W1 & & & \\
\hline Gobio gobio & & & & D1 & C1 & W1 & D1 & C1 & W1 \\
\hline Gobio uranoscopus & & & & $\mathrm{D} 2$ & $\mathrm{C} 3$ & W2 & & & \\
\hline Hucho hucho & & & & D1 & $\mathrm{C} 1$ & W1 & & & \\
\hline Lepomis gibbosus & D1 & C1 & W1 & & & & & & \\
\hline Leuciscus aspius & & & & D1 & C1 & W1 & D1 & C1 & W1 \\
\hline \multicolumn{10}{|l|}{ Neogobius fluviatilis } \\
\hline Perca fluviatilis & & & & & & & $\mathrm{D} 2$ & $\mathrm{C} 2$ & W2 \\
\hline Pseudorasbora parva & D1 & C1 & W1 & & & & & & \\
\hline Rhodeus amarus & D2 & C3 & W3 & D3 & $\mathrm{C} 2$ & $\mathrm{~W} 2$ & D5 & C3 & W4 \\
\hline \multicolumn{10}{|l|}{ Romanogobio kessleri } \\
\hline Romanogobio vladykovi & D3 & $\mathrm{C} 3$ & W3 & D5 & $\mathrm{C} 4$ & W5 & D5 & $\mathrm{C} 4$ & W5 \\
\hline Rutilus rutilus & D4 & C3 & W4 & & & & $\mathrm{D} 2$ & $\mathrm{C} 4$ & W3 \\
\hline \multicolumn{10}{|l|}{ Sabanejewia a. balcanica } \\
\hline \multicolumn{10}{|l|}{ Silurus glanis } \\
\hline Squalius cephalus & D6 & $\mathrm{C} 5$ & W6 & D4 & $\mathrm{C} 4$ & W4 & $\mathrm{D} 4$ & $\mathrm{C} 3$ & W4 \\
\hline Vimba vimba & & & & & & & $\mathrm{D} 2$ & $\mathrm{C} 2$ & W2 \\
\hline Zingel zingel & & & & & & & & & \\
\hline
\end{tabular}


Table 6: Ecological significance from three new designed SCIs in 2015.

\begin{tabular}{|c|c|c|c|c|c|c|c|c|c|}
\hline \multirow[t]{3}{*}{ Species } & \multicolumn{3}{|c|}{$\begin{array}{c}\text { Someşul Mare } \\
\text { River }\end{array}$} & \multicolumn{6}{|c|}{$\begin{array}{l}\text { Someș } \\
\text { River }\end{array}$} \\
\hline & \multirow[b]{2}{*}{$\begin{array}{c}\mathrm{D} \\
\text { class }\end{array}$} & \multirow[b]{2}{*}{$\begin{array}{c}\mathrm{C} \\
\text { class }\end{array}$} & \multirow[b]{2}{*}{$\begin{array}{c}\mathrm{W} \\
\text { class }\end{array}$} & \multicolumn{3}{|c|}{ Rona-Țicău } & \multicolumn{3}{|c|}{ Ardusat-border } \\
\hline & & & & $\begin{array}{c}\mathrm{D} \\
\text { class }\end{array}$ & $\begin{array}{c}\mathrm{C} \\
\text { class }\end{array}$ & $\begin{array}{c}\mathrm{W} \\
\text { class }\end{array}$ & $\begin{array}{c}\mathrm{D} \\
\text { class }\end{array}$ & $\begin{array}{c}\mathrm{C} \\
\text { class }\end{array}$ & $\begin{array}{c}\mathrm{W} \\
\text { class }\end{array}$ \\
\hline Alburnoides bipunctatus & D5 & $\mathrm{C} 3$ & W4 & D2 & $\mathrm{C} 2$ & W2 & D1 & $\mathrm{C} 2$ & W2 \\
\hline Alburnus alburnus & D6 & C5 & W6 & D4 & C5 & D5 & D6 & $\mathrm{C} 5$ & W6 \\
\hline Ballerus sapa & & & & & & & D1 & $\mathrm{C} 2$ & W1 \\
\hline \multicolumn{10}{|l|}{ Barbatula barbatula } \\
\hline Barbus barbus & D2 & $\mathrm{C} 4$ & W3 & D3 & $\mathrm{C} 4$ & W3 & D2 & $\mathrm{C} 3$ & W3 \\
\hline Barbus meridionalis & D5 & $\mathrm{C} 3$ & W4 & D3 & $\mathrm{C} 2$ & W3 & & & \\
\hline Carassius gibelio & & & & $\mathrm{D} 2$ & $\mathrm{C} 2$ & W2 & & & \\
\hline Chondrostoma nasus & $\mathrm{D} 2$ & C3 & W3 & D4 & $\mathrm{C} 4$ & W4 & & & \\
\hline \multicolumn{10}{|l|}{ Cobitis taenia } \\
\hline \multicolumn{10}{|l|}{ Cottus gobio } \\
\hline Gobio gobio & $\mathrm{D} 2$ & $\mathrm{C} 2$ & W2 & & & & & & \\
\hline \multicolumn{10}{|l|}{ Gobio uranoscopus } \\
\hline \multicolumn{10}{|l|}{ Hucho hucho } \\
\hline \multicolumn{10}{|l|}{ Lepomis gibbosus } \\
\hline Leuciscus aspius & D1 & C1 & W1 & D1 & C1 & W1 & $\mathrm{D} 2$ & C1 & W2 \\
\hline Neogobius fluviatilis & D1 & C1 & W1 & $\mathrm{D} 2$ & C3 & W3 & $\mathrm{D} 2$ & C3 & W2 \\
\hline Perca fluviatilis & & & & $\mathrm{D} 2$ & $\mathrm{C} 2$ & W2 & & & \\
\hline Pseudorasbora parva & & & & $\mathrm{D} 2$ & $\mathrm{C} 2$ & W2 & $\mathrm{D} 2$ & $\mathrm{C} 1$ & W1 \\
\hline Rhodeus amarus & D6 & $\mathrm{C} 3$ & W5 & D6 & $\mathrm{C} 5$ & W6 & D6 & $\mathrm{C} 4$ & W5 \\
\hline Romanogobio kessleri & D2 & $\mathrm{C} 4$ & W2 & D3 & $\mathrm{C} 2$ & W3 & & & \\
\hline Romanogobio vladykovi & D2 & $\mathrm{C} 2$ & W2 & D3 & C4 & W4 & D3 & C4 & W4 \\
\hline Rutilus rutilus & & & & $\mathrm{D} 1$ & $\mathrm{C} 1$ & W1 & $\mathrm{D} 2$ & $\mathrm{C} 3$ & W2 \\
\hline Sabanejewia a. balcanica & D1 & $\mathrm{C} 2$ & W2 & $\mathrm{D} 2$ & $\mathrm{C} 2$ & W2 & & & \\
\hline Silurus glanis & & & & & & & D1 & $\mathrm{C} 1$ & W1 \\
\hline Squalius cephalus & D6 & C4 & W5 & D6 & $\mathrm{C} 4$ & W5 & D4 & C3 & W4 \\
\hline Vimba vimba & D1 & C2 & W2 & D1 & $\mathrm{C} 1$ & W1 & D3 & $\mathrm{C} 3$ & W3 \\
\hline Zingel zingel & & & & & & & D1 & C1 & W1 \\
\hline
\end{tabular}


Regarding IBI all six studied SCIs has Scores between 57-67 which are included in II-IV Evaluation integrity class, means moderate-good to excellent-good appreciation in fish ecosystem (Tab. 7).

Table 7: IBI results for fish fauna from six SCIs from Someș and Mureș rivers.

\begin{tabular}{|c|c|c|c|c|c|c|}
\hline \multirow{2}{*}{ 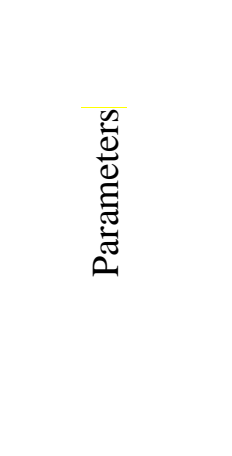 } & \multicolumn{2}{|c|}{ Someșul Mic } & Someșul Mare & Someșul Unit & \multicolumn{2}{|r|}{ Mureș } \\
\hline & 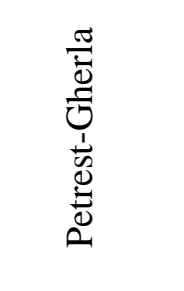 & 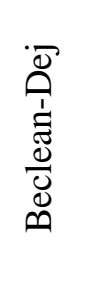 & 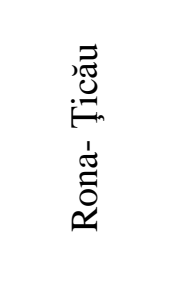 & 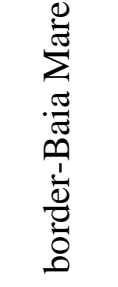 & 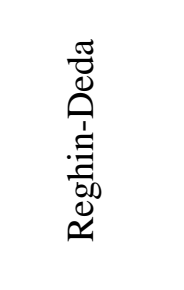 & 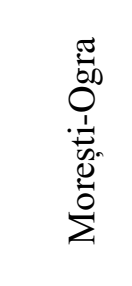 \\
\hline 1. & 5 & 5 & 5 & 5 & 5 & 5 \\
\hline 2. & 5 & 5 & 5 & 5 & 5 & 5 \\
\hline 3. & 1 & 1 & 1 & 1 & 1 & 1 \\
\hline 4. & 3 & 3 & 3 & 3 & 5 & 3 \\
\hline 5. & 5 & 5 & 5 & 5 & 5 & 5 \\
\hline 6. & 1 & 5 & 3 & 3 & 5 & 5 \\
\hline 7. & 3 & 3 & 3 & 1 & 5 & 5 \\
\hline 8. & 5 & 5 & 5 & 5 & 5 & 5 \\
\hline 9. & 5 & 5 & 5 & 5 & 5 & 5 \\
\hline 10. & 3 & 3 & 3 & 3 & 3 & 3 \\
\hline 11. & 5 & 3 & 3 & 5 & 3 & 3 \\
\hline 12. & 3 & 5 & 5 & 5 & 5 & 3 \\
\hline 13. & 3 & 3 & 3 & 5 & 5 & 3 \\
\hline 14. & 5 & 5 & 5 & 5 & 5 & 5 \\
\hline 15. & 5 & 5 & 5 & 5 & 5 & 5 \\
\hline Score & 57 & 61 & 59 & 61 & 67 & 61 \\
\hline $\begin{array}{l}\text { Evaluation } \\
\text { integrity } \\
\text { class }\end{array}$ & IV & III & IV & III & II & III \\
\hline Appreciations & $\begin{array}{l}\text { Moderate- } \\
\text { good }\end{array}$ & good & $\begin{array}{l}\text { Moderate- } \\
\text { good }\end{array}$ & good & $\begin{array}{l}\text { Excellent- } \\
\text { good }\end{array}$ & good \\
\hline
\end{tabular}


Mureș River (ROSCI0367 Mureș between Morești-Ogra) - In this extended SCI (Morești-Ogra) we have identified 12 fish species including four ones community interest species present, none in addition to the Standard Form N2000 List, but three fish species of community interest (S. aurata, G. kessleri and Z. streber) did not find. Main and characteristic or complementary species, dominated in abundance are Alburnus alburnus, Rhodeus amarus, G. albipinatus, Squalius cephalus (Fig. 2; Tab. 5). This sector of Mureș River is affected mostly by industrial and domestic pollution, but in researches time period with low intensity.

Mureș River (ROSCI0368 Mureș between Reghin-Deda) - In this extended SCI we have identified 15 fish species including nine ones community interest species, with new captured species Hucho hucho (Fig. 8) and Cottus gobio compared with Standard Form N2000 List, but was not found in studied period Gobio kessleri. Main and characteristic or complementary species dominant in abundance are Alburnoides bipunctatus, Gobio albinnatus, Barbus meridionalis and Chondrostoma nasus (Fig. 3; Tab. 5). In this sector of Mureș River low human impact is present: angling, tourists and rocks exploitations.

Someșul Mic River (ROSCI0394 Someșul Mic) (sampling near Gherla and Mintiu Gherlii localities) - It is an extended SCI with 11 captured fish species including four ones community interest, with G. albipinnatus and B. meridionalis new added in Standard Form N2000 List, but C. taenia and G. kessleri missing in captures in studied period. Main and characteristic or complementary species dominant in abundance are B. meridionalis, $S$. cephalus and $R$. rutilus (Fig. 4; Tab. 5). Someșul Mic River is affected mostly by industrial, agricultural and domestic pollution, also dams, but actual with medium intensity.

Someș River (ROSCI0435 Someșul Inferior) - New designed SCI on Someș River between Ardusat locality and Romania-Hungary border, designated for 14 fish species including four ones of community interest. Main, characteristic and complementary or complementary species are Alburnus alburnus, R. amarus, G. albipinatus and S. cephalus (Fig. 7; Tab. 6). Here were found many individuals of Neogobius fluviatilis (Fig. 9) new recorded species in Someș River first time recorded in Someș Unit, upstream between JibouDej localities in 2014 (Cocan et al., 2014). Someș River is affected mostly by industrial, agricultural and domestic pollution, also ballast exploitation, but actual with medium intensity.

Someș River (ROSCI0436 Someș between Rona-Țicău) - It is new designed SCI for fish fauna with 17 fish species including six ones community interest, main and characteristic or complementary species dominant in abundance are R. amarus, S. cephalus and A. alburnus (Fig. 6; Tab. 6). Also in this sector of Someș River was found many individuals of Neogobius fluviatilis, new recorded gobies in river (confirming Cocan et al. (2014) records). This part of Someș River is affected mostly by industrial and domestic pollution, also forestry exploitation and angling, but actual with low intensity.

Someșul Mare River (ROSCI0394 Someșul between Mica-Beclean) - Is new SCI designed for fish fauna with 14 fish species including six ones community interest, main and characteristic or complementary species dominant in abundance are R. amarus, S. cephalus, A. alburnus, A. bipunctatus and B. meridionalis (Fig. 5; Tab. 6). Very important is the presence of some individuals of $N$. fluviatilis in Someșul Mare River between Mica-Beclean, new recorded species in this tributary of Someș River, not present in fish list of Bănărescu et al. (1999). This species booming population in all Danube Basin including Someș tributaries, first record in united Someș Unit (Cocan et al., 2014). Someșul Mare River is affected mostly by ballast exploitation follow by agricultural and domestic pollution, also angling and fish expansion of $N$. fluviatilis competitor to other fish species, but with low intensity. 


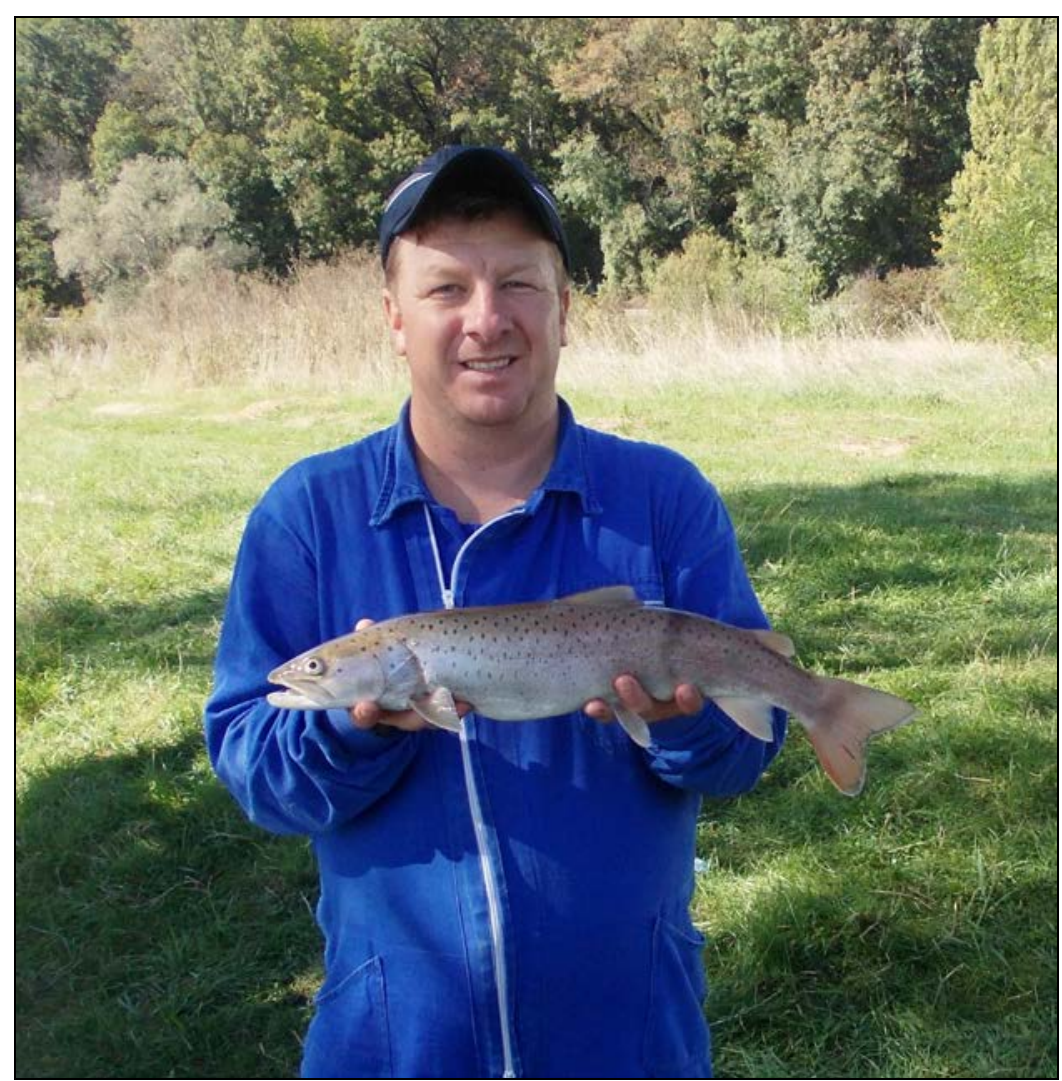

Figure 8: Hucho hucho critical endangered fish species captured in Mureș River, near Brâncovenești locality (two individuals), quickly released in water.

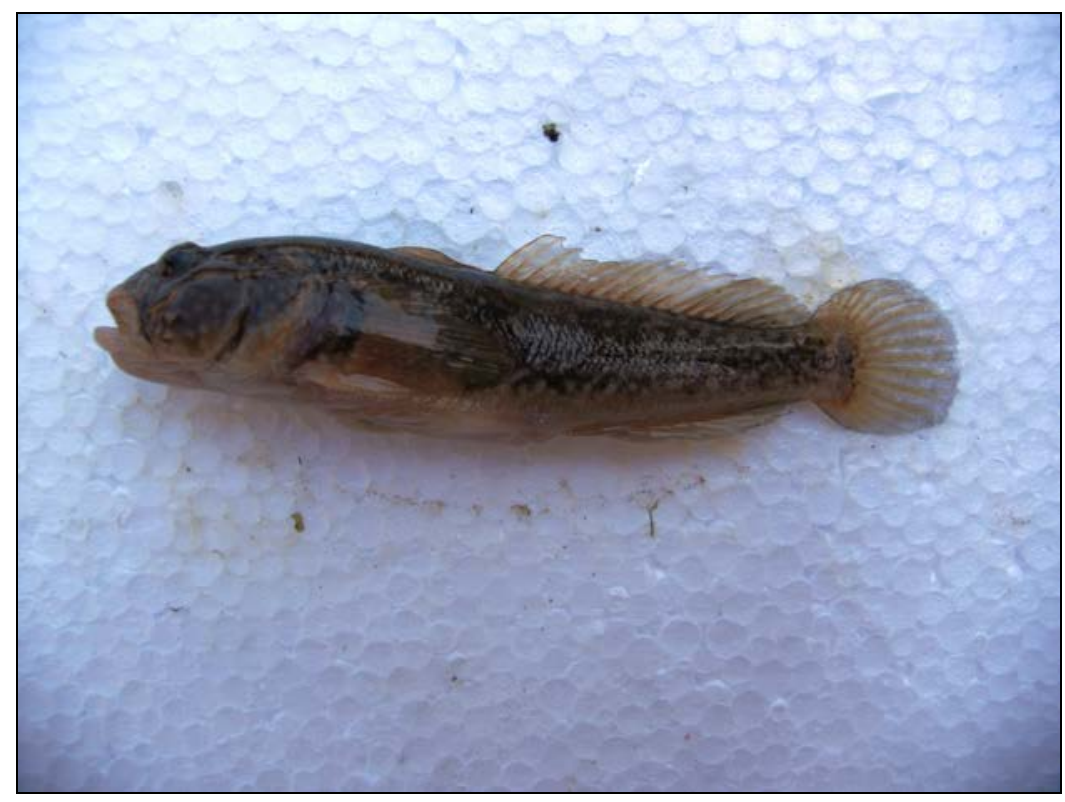

Figure 9: New record of Neogobius fluviatilis in Someșul Mare River, upstream Dej locality (10 individuals near Uriu locality), in expansion in all Danube Basin. 


\section{CONCLUSIONS}

In 2015 were designed three new SCI for fish fauna and other three SCI were extended, all six for solving IN MOD stage of Aspius aspius, Gobio albipinnatus and Rhodeus amarus related with mammal Lutra lutra.

This three IN MOD fish species have stable population with favourable development conditions in all six studied SCIs.

In these six investigated SCIs were captured 27 fish species including 11 ones of community interest with three more species (Hucho hucho, Cottus gobio and Zingel zingel) that was in Standard Form N2000 List, but Zingel streber missed from capture in 2015.

Main and characteristic or complementary fish species dominant in abundance are Alburnus alburnus in plain area of studied sectors and Alburnoides bipunctatus in submontain area, followed by Squalius cephalus, Rhodeus amarus, Gobio albipinnatus and Barbus meridionalis with some differences between sites.

The most important fish species captured was two individuals of Hucho hucho in Mureș River, near Brâncovenești locality, very rare species critical endangered, its presences is encouraging.

Neogobius fluviatilis (monkey goby) was first recorded by us far upstream in Someșul Mare than its first record in Someș (2014), which indicates its expansion in Someș River system. Its expansion is the same as in all Danube Basin.

Dams, river rocks extraction, ballast and forestry exploitation or agricultural, industrial, touristic, urban pollution and expansion of competitor Neogobius fluviatilis, all of these factors have negative impact in Mureș and Someș rivers, disturbing pristine/foremost fish conditions, demaging initial wildlife habitats.

Advanced investigations in time (more seasons) and space (more sampling sites) for SCIs evolution and for finding very rare species are necessary in future. 
ACKNOWLEDGEMENTS

This work was funded by Romanian Ministry of Environment, Water and Forests through "Consolidation the Natura 2000 network" project evaluation of community interest species in new design or extended Romanian SCIs, on this way we send our gratitude. We thanks also to DDNIRD fisherman (Iosif N.) and driver (Caramangiu G.) for their help in all weather conditions of field work of tent accommodation in 2015, also for the German abstract translation thanks to Mr. Suciu R. 


\section{REFERENCES}

1. Antal L., Halasi-Kovács B. and Nagy S. A., 2013 - Changes in fish assemblage in the Hungarian section of river Someş after a massive cyanide and heavy metal pollution, North-Western Journal of Zoology, 9, 1, 131-138.

2. Battes K. W., 1991 - Acvacultură, prezent și perspectivă, Ecologia et Aquacultura Limnica, 2, 11, 337-425. (in Romanian)

3. Bănărescu P., 1964 - Fauna R. P. R., XIII, Pisces-Osteichthyes, Edit. Academiei R. P. R., Bucureşti, 962. (in Romanian)

4. Bănărescu P., 1994 - The present-day conservation status of the freshwater fish fauna of Romania, Ocrotirea naturii şi mediului inconjurător, Academia Română, 38, 1, $1-16$.

5. Bănărescu P., 2004 - Situaţia actuală a ihtiofaunei de apă dulce a României sub aspect faunistic, taxonomic şi al protecţiei, Studia Universitatis Vasile Goldiş Arad, Seria Ştiinţele Vieţii, 14, 4349. (in Romanian)

6. Bănărescu P., Telcean I., Nalbant T., Harka Á. and Ciobanu M., 1999 - The fish fauna of the river Someș Basin, 249-266, in Sárkány-Kiss A. and Hamar J. (eds), Tiscia monograph series, The Someș River valley. A study of the geography, hydrobiology and ecology of the river system and its environment, Târgu-Mureș.

7. Botnariuc N. and Vădineanu A., 1982 - Ecologie, Edit. Didactică și Pedagogică, Bucureşti, 359-418 (Anexele 1-12). (in Romanian)

8. Brînzan T. (ed.), 2013 - Catalogul habitatelor, speciilor şi siturilor Natura 2000 din România, Edit. Fundaţia Centru Naţional pentru Dezvoltare Durabilă - Bucureşti, 784. (in Romanian)

9. Cocan D., Mireşan V., Oţel V., Păpuc T., Laţiu C., Coşier V., Constantinescu R. and Răducu C., 2014 - First Record of the Pontian Monkey Goby Neogobius fluviatilis (Pallas, 1814) in the Someş River, Transylvania - Romania, AACL Bioflux ProEnvironment, 7, 240-246.

10. Curtean-Bănăduc A. and Bănăduc D., Bucşa C., 2007 - Watersheds Management (Transylvania, Romania), Implications, risks, solutions, Strategies to enhance environmental Security in transition countries, NATO Science for Peace and Security Series C-Environmental Security, Springer, 225-238, DOI: 10.1007/978-1-4020-5996-4_17, ISSN 1971-4668, ISBN 978-1-40205994-0.

11. Curtean-Bănăduc A., Bănăduc D., Burcea A., Berg V., Lyche J. L. and Gheorghe L. M., 2017 Persistent organic pollutants in Mureş Watershed, in The impact of persistent organic pollutants on freshwater ecosystems and human health, Curtean-Bănăduc A. (ed.), 119-154.

12. Froese R. and Pauly D. (eds), 2016 - FishBase, World Wide Web electronic publication, www.fishbase.org, version (09/2016).

13. Gomoiu M. T. and Skolka M., 2001 - Ecologie. Metodologii pentru studii ecologice, “Ovidius” University Press, Constanţa, ISBN 973-614-001-6, 175. (in Romanian)

14. Karr J. R., Fausch K. D., Angermeier P. L., Yant P. R. and Schlosser I. J., 1986 - Assessing biological integrity in running waters: a method and its rationale, Illinois Natural History Survey, Special Publication, 5, 28.

15. Köhler H. R., Triebskorn R. and Sandu C., 2005 - Monitoring pollution in river Mureş, Bulletin of the International Association for Danube Research, 11, 1-6.

16. Köhler H.-R., Sandu C., Scheil V., Nagy-Petrică E. M., Segner H., Telcean I., Stan G. and Triebskorn R., 2007 - Monitoring Pollution in river Mureş, Romania, Part III: Biochemical Effect Markers in Fish and Integrative Reflection, Environmental Monitoring and Assessment, 127, 47-54.

17. Kottelat M., 1997 - European freshwater fishes: a heuristic checklist of the freshwater fishes of Europe (exclusive of former USSR), with an introduction for non-systematists and comments on nomenclature and conservation, Biologia (Bratislava), 52, 5, 1-271. 
18. Kottelat M. and Freyhof J., 2007 - Handbook of European Freshwater Fishes, Kottelat, Cornol, Switzerland and Freyhof, Berlin, Germany, 464.

19. Miller D. L., 1985 - Technological thinking: its impact on environmental management, Environmental Management, 9, 179-190.

20. Mühlenberg M., 1993 - Freilandökologie, 3, überarbeitete Auflage, UTB für Wissenschaft 595, Quelle and Meyer, Heidelberg, Wiesbaden, 512. (in German)

21. Nalbant T., 1993 - Some problems in the systematics of the genus Cobitis and its relatives (Pisces, Ostariophysi, Cobitidae), Revue Roumaine de Biologue, Serie Biologi Animale, 38, 2, 101-110.

22. Nalbant T., 1994 - Studies on loaches (Pisces: Ostariophysi: Cobitidae), I, An evaluation of the valid genera of Cobitinae, Travaux du Muséum National d'Histoire Naturelle "Grigore Antipa”, XXXIV, 375-380.

23. Nalbant T., 1995 - Fish of the Mureş River: Systematics and Ecology, 225-234, in Sárkány-Kiss A. and Hamar J. (eds), Tiscia monograph series, The Mureș River valley. A study of the geography, hydrobiology and ecology of the river and its environment, TârguMureș.

24. Năstase A. and Oțel V., 2016 - Researches on the fish fauna in some SCIs Natura 2000 from Romania, AACL Bioflux, 9, 3, 527-540.

25. Odum E. P., 1975 - Ecology: The link Between the Natural and the Social Sciences, Second Edition, A Holt International Edition, 244.

26. Oţel V. and Năstase A., 2010 - Researches on ichthyofauna in the Natura 2000 sites from Banat (Romania), Scientific Annals of the Danube Delta Institute, 16, Tulcea, Romania, 33-38.

27. Sandu C., Bloesch J. and Coman A., 2008 - Water pollution in the Mureş Catchment and its impact on the aquatic communities (Romania), Transylvanian Review of Systematical and Ecological Research, 6, The Wetlands Diversity, 97-108.

28. Sârbu I. and Benedek A. M., 2004 - Ecologie practică, Edit. Universitatea „Lucian Blaga” din Sibiu, 260. (in Romanian)

29. Schwerdtfeger F., 1975 - Ökologie der Tiere Band 3: Synökologie, Paul Parey Verlag, Hamburg, Berlin, 451. (in German)

30. Şindrilariu P. D., Bacalbaşa-Dobrovici N., Freyhof J. and Wolter C., 2002 - The juvenile fish community of the Lower Danube and Danube Delta, Brezeanu G. and R. Ştiucă (eds), International Association for Danube Research, 34, 517-526.

31. Tatole V., Iftime A., Stan M., Iorgu E.-I. and Oţel V., 2009 - Speciile de animale Natura 2000 din România, Bucureşti, 60-86.

32. *, 1992 - Directiva Consiliului Uniunii Europene nr. 92/43/1992 privind conservarea habitatelor naturale, faunei şi florei sălbatice (Habitats Directive) (Council Directive 92/43/EEC of 21 May 1992 on the conservation of natural habitats and wild fauna and flora) in Official Journal of the European Union, L206, 7-50, Luxembourg.

33. **, 2007 - Ordonanţa de Urgenţă a Guvernului nr. 57 din 20 iunie 2007 privind regimul ariilor naturale protejate, conservarea habitatelor naturale, a florei şi faunei sălbatice, in Monitorul Oficial al României, Partea I, anul 175 (XIX), 442 (29 iunie 2007), 1-32, Bucureşti, Romania. (in Romanian)

34. ***, 2011 - Legea nr. 49 din 7 aprilie 2011 pentru aprobarea Ordonanţei de urgenţă a Guvernului nr. 57/2007 privind regimul ariilor naturale protejate, conservarea habitatelor natural, a florei şi faunei sălbatice, in Monitorul Oficial al României, Partea I, anul 179 (XXIII), 262 (13 aprilie 2011), 2-12, Bucureşti, România. (in Romanian) 
35. ****, 2011a - Ordin al Ministrului Mediului şi Pădurilor nr. 2387/29 IX 2011 pentru modificarea Ordinului Ministrului Mediului şi Dezvoltării Durabile nr. 1964/13 XII 2007 privind instituirea regimului de arie naturală protejată a siturilor de importanţă comunitară, ca parte integrantă a rețelei ecologice europene Natura 2000 în România, in Monitorul Oficial al României, Partea I, anul 179 (XXIII), nr. 864 (din 29 noiembrie 2011), 11, Bucureşti, România. (in Romanian) 